\title{
LINEAR MODELS AND SET THEORY
}

\author{
Santiago Jockwich Martinez ${ }^{1} \&$ Giorgio Venturi ${ }^{2}$
}

\begin{abstract}
In this paper we review the approach to algebra-valued models and we introduce an infinite class of natural models for paraconsistent set theory. We then present several paraconsistent set theories based on Logics of Formal Inconsistency (LFI), known in the literature as LFI-set theories, and we show that there is no natural model that validates these systems. We therefore suggest to abandon these theories.
\end{abstract}

\section{Introduction}

There are two possible approaches to paraconsistent set theory. The first one, that we may call Cantorian, stems from the recognition that the troubles caused by the foundational paradoxes is not ascribable to the basic principles of the theory but to the underlying logic. This approach calls for a revision of the logic and proposes new logical tools to escape the triviality that originates from set-theoretical antinomies. Generally, this kind of approach aims at formalizing set theory by means of two simple principles: Extensionality and unrestricted Comprehension. Examples of these theories can be found in [3], [14], and [15]. While the proponents of these paraconsistent set theories consider their systems to be closer in spirit to Cantor's theory - and to the naïve idea that to every well-defined property corresponds a set-however their justification suffer of the same weakness that an intuitionist axiomatic system suffers with respect to Brower's intuitionism: how to consider appropriate the use of axioms and deductive rules for regimenting theories that in the intentions of their creators tended to escape formalization? In other words, how to conciliate the axiomatic method and a naïve approach to Cantor's theory of transfinite numbers?

In direct opposition to the Cantorian approach we may find what we may called Zermelian paraconsistent set theories. These are the axiomatic systems that, using an underlying paraconsistent logic, accept as a fundamental step in the development of set theory the axiomatization that Zermelo proposed in the 1908 paper Investigations in the foundations of set theory I. From this perspective, classical ZFC captures only partially the universe of set theory, while a paraconsistent approach is justified in that it offers a broader view of a theory which includes consistent and inconsistent totalities - to use a Cantorian phrasing. To put it more emphatically: there are more things in heaven and earth than are dreamt of in ZFC. Consequently, a Zermelian approach proposes axiomatic systems as close as possible to classical ZFC, but where the logical connective receive, instead, a paraconsistent interpretation. Example of these theories may be found in [8], [9], and [5]. Although both approaches have their pros and cons, we consider the Zermelian approach closer to set-theoretical practice.

It is undeniable that, from 1908 on, the axiomatization of set theory brought a clearer picture of the universe of all sets - after decades of works and the efforts of mathematicians

\footnotetext{
${ }^{1}$ University of Campinas, UNICAMP. Email: santijoxi@hotmail.com.

${ }^{2}$ University of Campinas, UNICAMP. Email: gio.venturi@gmail.com.

DOI : 10.21452/LnA_serie_n_v01_book_seminario-logica-no-aviao-2013-2018_martinez-venturi_p.09-21
} 
of the caliber of Zermelo, Fraenkel, Skolem, Von Neumann, and Gödel - in terms of the cumulative structure of $\mathbf{V}$. Moreover, on the philosophical side, Boolos [2] argued for a deep conceptual connection between a cumulative hierarchy structure, the iterative conception of set, and ZFC. For this reason, we believe that the Zermelian approach should not only be restricted to a system of axioms, but should also consider its semantic interpretation, in terms of a suitable cumulative hierarchy. This idea has been made concrete in [9], using algebra-valued models; i.e. a generalization of Boolean-valued models to structures whose underlying algebra is not necessarily Boolean. In [9], the authors introduced an algebra-valued model $\mathbf{V}^{\left(\mathbb{P S}_{3}, *\right)}$, based on the paraconsistent algebra $\left(\mathbb{P S}_{3}, *\right)$, first introduced in [10]. The approach of [9] has been recently extended in [12] to produce an infinite class of models, called linear, which include the model $\mathbf{V}^{\left(\mathbb{P S}_{3}, *\right)}$ as a special case.

In this paper we investigate wether linear models are suitable structures for the set theories developed in [5]. The reason for asking this question is twofold. First, the linear models are the only know models for paraconsistent Zermelian set theories. Moreover, being the result of algebra-valued constructions, linear models can be seen as natural models for the Zermelian approach, because of their cumulative hierarchy structure. Second, and mostly, the algebra $\left(\mathbb{P S}_{3}, *\right)$ is a Logic of Formal Inconsistency, as shown in [7]. Therefore it is natural to interpret the axioms of these axiomatic systems in a model like $\mathbf{V}^{\left(\mathrm{PS}_{3}, *\right)}$, which is based on the same logical background.

In the next pages we will show that the set theories developed in [5] are not valid in any linear model. This will therefore motivate the rejection of these theories. More precisely we will show that there is a deep conceptual issue in the formulation of these axiomatic systems, since they fall short of capturing inconsistent totalities. In short, these systems do not comply with the task for which they have been created; either they get a harmless interpretation of paraconsistency (i.e. classical ZF), or they are just false in the natural models of Zermelian paraconsistent set theories.

The article is structured as follows. In $\S 2$ we introduce the Boolean-valued technique for constructing models of set theory. In $\S 3$ we introduce the linear models for paraconsistent set theory. In $\S 4$ we introduce a family of Zermelian paraconsistent set theories based on the language of LFIs - as presented in [5] — and we show that linear models do not validate them. We conclude offering reasons to abandon these set theories.

\section{Boolean valued models}

The Boolean-valued model technique represents an alternative, algebraic, presentation of the forcing method. Forcing was invented by Paul Cohen [6] and is now considered the cornerstone of contemporary set theory. It is a tool for producing relative consistency results, that has been used to prove the independence of $\mathrm{CH}$ from the axioms of ZFC.

To recall the main ideas and to fix notation we briefly introduce the Boolean-valued model construction of a model of set theory. ${ }^{3}$ Given a complete Boolean algebra $\mathbb{B}$, a Boolean-valued structure is defined by transfinite recursion:

$\mathbf{V}_{\alpha}^{\mathbb{B}}=\left\{x \mid x\right.$ is a function and $\operatorname{ran}(x) \subseteq \mathbb{B}$ and there is $\zeta<\alpha$ with $\left.\operatorname{dom}(x) \subseteq \mathbf{V}_{\zeta}^{\mathbb{B}}\right\}$ and

$$
\mathbf{V}^{\mathbb{B}}=\left\{x \mid \exists \alpha\left(x \in \mathbf{V}_{\alpha}^{\mathbb{B}}\right)\right\}
$$

\footnotetext{
${ }^{3}$ For reasons of space, we do not define notions that are standard in the literature. For a broader and more complete presentation, we defer the interested reader to [1].
} 
Extending the language of set theory with constants for every element of $\mathbf{V}^{\mathbb{B}}$, we can define a new language $\mathcal{L}^{\mathrm{B}}$, as common in model theory, that allows one to talk about the Boolean valued structure $\mathbf{V}^{\mathbb{B}}$, by means of what are called $\mathbb{B}$-sentences. We can therefore generalize the notion of satisfaction. Indeed, it is possible to define a B-evaluation function that assigns to every $\mathbb{B}$-sentence $\sigma$, its Boolean truth value $\llbracket \sigma \rrbracket \in \mathbb{B}^{4}$ Hence, whenever $\llbracket \varphi \rrbracket=1_{B}$ (that is, the maximal element of the complete Boolean algebra $\mathbb{B}$ ), then we say that $\varphi$ is valid in $\mathbf{V}^{\mathbb{B}}$. The definition the $\mathbb{B}$-evaluation function not only resembles closely that of a truth definition, but, similarly, it cannot be fully defined within ZFC, because, as in the case of the truth predicate, the collection of all ordered pairs $\left(\sigma, \llbracket \sigma \rrbracket^{\mathbb{B}}\right)$ is not a definable class in ZFC. ${ }^{5}$

The Boolean-valued structures so constructed are non-standard in a very strong sense. Indeed, the interpretations of the basic notions of equality and membership have an ad hoc non-standard character. Given a complete Boolean algebra $\mathbb{B}$ and $u, v \in \mathbf{V}^{\mathbb{B}}$,

$$
\llbracket u \in v \rrbracket=\bigvee_{y \in \operatorname{dom}(v)}(v(y) \wedge \llbracket u=y \rrbracket)
$$

and

$$
\llbracket u=v \rrbracket=\bigwedge_{x \in \operatorname{dom}(u)}(u(x) \Rightarrow \llbracket x \in v \rrbracket) \wedge \bigwedge_{y \in \operatorname{dom}(v)}(v(y) \Rightarrow \llbracket y \in u \rrbracket) .
$$

Notice that the above evaluations are inter-defined by a simultaneous recursion and in terms of the algebraic structure of the Boolean algebra. On the other hand, the other logical connective are interpreted in the standard way.

$$
\begin{gathered}
\llbracket \perp \rrbracket^{\mathbb{B}}=0_{B}, \\
\llbracket \varphi \wedge \psi \rrbracket^{\mathbb{B}}=\llbracket \varphi \rrbracket^{\mathbb{B}} \wedge_{\mathbb{B}} \llbracket \psi \rrbracket^{\mathbb{B}}, \\
\llbracket \varphi \vee \psi \rrbracket^{\mathbb{B}}=\llbracket \varphi \rrbracket^{\mathbb{B}} \vee_{\mathbb{B}} \llbracket \psi \rrbracket^{\mathbb{B}}, \\
\llbracket \varphi \rightarrow \psi \rrbracket^{\mathbb{B}}=\llbracket \varphi \rrbracket^{\mathbb{B}} \Rightarrow_{\mathbb{B}} \llbracket \psi \rrbracket^{\mathbb{B}}, \\
\llbracket \exists x \varphi(x) \rrbracket^{\mathbb{B}}=\vee_{u \in V^{\mathbb{B}}} \llbracket \varphi(u) \rrbracket^{\mathbb{B}}, \text { and } \\
\llbracket \forall x \varphi(x) \rrbracket^{\mathbb{B}}=\wedge_{u \in V^{\mathbb{B}}} \llbracket \varphi(u) \rrbracket^{\mathbb{B}},
\end{gathered}
$$

where on the left side of the equality are displayed logical connectives, while on the right side the operations of the algebra $\mathbb{B}$, and the negation is defined in terms of implication and $\perp$.

The interest of this construction lays in the fact that, due to the properties of Boolean algebras, all axioms of ZFC are valid in $\mathrm{V}^{\mathbb{B}}$, that is, if $\sigma$ is an axiom of ZFC, then $\llbracket \sigma \rrbracket^{\mathbb{B}}=1_{B}$. On the other hand, if $\llbracket \sigma \rrbracket \neq 0_{B}$, we can say that $\sigma$ is consistent with ZFC. Moreover, the versatility of this method consists in the possibility to obtain different consistency results just by changing the underlying algebra and thus constructing different models.

\footnotetext{
${ }^{4}$ Remember that $0_{B}$ and $1_{B}$, respectively the smallest and largest elements of a Boolean algebra $\mathbb{B}$, may be interpreted as falsehood and truth; hence, all other elements of the algebra have the intuitive meaning of intermediate truth values.

${ }^{5}$ However, notice that, due to the definability in the ground model of the forcing relation, we have that, fixing a formula $\varphi$, the collection $\left\{\left(\tau, \varphi(\tau) \mid \tau \in \mathbf{V}^{\mathbb{B}}\right\}\right.$ is a class definable in $\mathbf{V}^{\mathbb{B}}$.
} 


\section{Models for Zermelian set theories}

Definition 1. We call a poset $(A ; \leqslant)$ a meet semilattice if every two elements $x, y \in A$ have an infimum, denoted by $x \wedge y$. If there also exists a supremum, $x \vee y$, for any two elements $x, y \in A$, then $(A ; \leqslant)$ is a lattice. We say that $(A ; \leqslant)$ is a bounded lattice if it is a lattice that has a greatest element $1_{A}$ and a least element $0_{A}$. A lattice $(A ; \leqslant)$ is complete if the supremum $\bigvee X$ and the infimum $\wedge X$ exist for every $X \subseteq A$. A lattice is called distributive if it satisfies the distributivity law, that is, $x \wedge(y \vee z)=(x \wedge y) \vee(x \wedge z)$ for all $x, y, z$ in its universe.

In what follows, by an algebra we mean a complete bounded distributive lattice. A first step in the construction of natural models for Zermelian paraconsistent set theories has been undertaken in [9], modifying the underlying algebra of a Boolean-valued model construction. The authors of [9] individuated a specific class of algebra suited for this purpose: the reasonable implicative algebras (ri-algebras henceforth). Following [9], an algebra, in order to be a ri-algebra needs to have an operation $\Rightarrow$ that satisfies the following properties: 6

$\mathbf{P} 1(x \wedge y) \leqslant z$ implies $x \leqslant(y \Rightarrow z)$

P2 $y \leqslant z$ implies $(x \Rightarrow y) \leqslant(x \Rightarrow z)$

P3 $y \leqslant z$ implies $(z \Rightarrow x) \leqslant(y \Rightarrow x)$

$\mathbf{P} 4((x \wedge y) \Rightarrow z)=(x \Rightarrow(y \Rightarrow z))$

But a further condition (or better a schema of conditions for every $\varphi$ a negation free fromula) is then required, in order validate all axioms of ZF.

$$
\llbracket \forall x \varphi(x) \rrbracket^{\mathbb{A}}=\bigwedge_{x \in \operatorname{dom}(u)}\left(u(x) \Rightarrow \llbracket \varphi(x) \rrbracket^{\mathbb{A}}\right) \quad\left(\mathcal{B} \mathcal{Q}_{\varphi}\right)
$$

THEOREM 2. ([9]) Any ri-algebra $\mathbb{A}$ that satisfies $\mathcal{B Q}_{\varphi}$, for any negation free formula $\varphi$, is such that the model $\mathbf{V}^{\mathbb{A}}$ validates the negation free fragment of $Z F$.

In [9] we find an example of an algebra, called $\mathbb{P S}_{3}$, for which the hypotheses - and thus the conclusion - of Theorem 2 hold. Thus, $\mathbf{V}^{\mathbb{P S}_{3}}$ validates all axioms of ZF, with the axiom schemata restricted to negation free formulas.

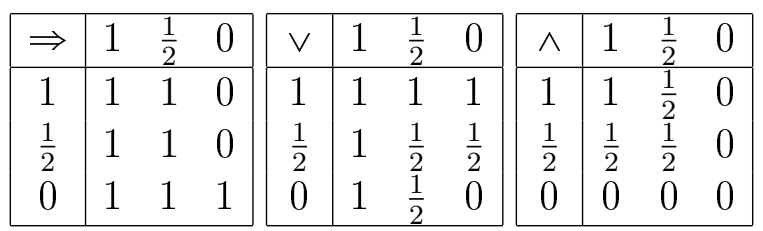

Figure 1. Connectives for $\mathrm{PS}_{3}$

${ }^{6}$ A caveat for the reader: by an ri-algebra we mean what in [9] was called reasonable deductive implication algebra. We slightly changed the terminology for notational and expository convenience. We hope not to confuse the well-informed reader. 
The main result of [9] consisted in showing that it is also possible to add a paraconsistent negation ${ }^{*}$, defined by $1^{*}=0, \frac{1}{2}^{*}=\frac{1}{2}$ and $0^{*}=1$, in such a way that $\mathbf{V}^{\left(\mathbb{P S}_{3}{ }^{*}\right)}$ is a paraconsistent model of the negation free fragment of ZF (which will be abbraviated as NFF-ZF). More recently, in [12], this approach has been extended to show that $\mathbf{V}^{\left(\mathbb{P S}_{3},{ }^{*}\right)}$ is only one element of a uniformly defined class of models for paraconsistent NFF-ZF.

DeFinition 3. By $\mathfrak{V}$ we indicate the class of linear algebras of the form $\mathbb{L}=(L, \wedge, \vee, \neg, D, 0,1)$, where $\neg x=\max \{y: x \wedge y=0\}$ exists for every $x \in L$ as well as $D x=\min \{y \in L: x \vee y=1\}$ exists for every $x \in L$, such that the base set is order-isomorphic to an ordinal. By an $\mathfrak{V}$-algebra we mean a member of $\mathfrak{V}$.

THEOREM 4. ([12]) Given an $\mathfrak{V}$-algebra $\mathbb{L}$, it is possible to define an implication that not only makes it an ri-algebras, but that also satisfies $\mathcal{B Q}_{\varphi}$, for any negation free formula $\varphi$. Moreover, it is possible to define a paraconsistent negation in such a way that $\mathbf{V}^{\mathbb{L}}$ is a model of paraconsistent $\mathrm{ZF}$.

We refer to an $\mathfrak{V}$-algebra also as $\mathbb{L}=(L ; \wedge, \vee, \rightsquigarrow, *)$, displaying the two operations $\rightsquigarrow$ and $*$ of Theorem 4 . These operations are used to interpret the implication and the negation from the language of set theory. For this reason we call these algebraic operations, respectively, implication and negation.

LEMMA 5. ([12]) Let $\mathbb{L}$ be a linear algebra of the form $\mathbb{L}=(L ; \wedge, \vee, \rightsquigarrow, *)$. When $|\mathbb{L}|=2$ we get that $\mathbf{V}^{\mathbb{L}}=\mathbf{V}^{\mathbb{B}_{2}}$, where $\mathbb{B}_{2}$ is the two-elements Boolean algebra, while when $|\mathbb{L}|=3$ we get that $\mathbf{V}^{\mathbb{L}}=\mathbf{V}^{\left(\mathbb{P S}_{3},{ }^{*}\right)}$.

The names negation and implication for the characteristic operations of linear algebras are well justified by the the next two lemmas that show their proximity to the classical case.

LEMMA 6. ([12]) The negation * of a linear algebra $\mathbb{L}$ maps each intermediate value of the base set to itself, that is for all $a \in L$ such that $0 \neq a \neq 1$ we have $a^{*}=a$. Besides that $0^{*}=1$ and $1^{*}=0$.

Lemma 7. ([12]) For any $\mathbb{L} \in \mathfrak{V}$ and for any $x, y \in \mathbf{V}^{\mathbb{L}}$ the following holds: $\llbracket \varphi(x) \rightsquigarrow$ $\psi(y) \rrbracket=0$ if and only if $\llbracket \varphi(x) \rrbracket \neq 0$ and $\llbracket \psi(y) \rrbracket=0$.

\section{Logics of Formal Inconsistency and their set theories}

In this section we briefly introduce the Zermelian set theories we will discuss in this paper and the logics on which they are based.

4.1 Logics of Formal Inconsistency. The LFIs form a family of paraconsistent logics that extend the syntactic approach of Da Costa to paraconsitency, by expressing consistency at the meta-linguisitic level by means of a unary operator which extends the logical language. These logics where first introduced by João Marcos in his doctoral thesis [11]. ${ }^{7}$ More syntactically, we can define these logics as those which satisfy the following two conditions:

(1) There is a set of formulas $\Gamma$ and formulas $\alpha$ and $\beta$ such that $\Gamma \cup\{\alpha, \neg \alpha\} \not \forall \beta$.

\footnotetext{
${ }^{7}$ For an up to date presentation of LFI see [4].
} 
(2) Given a formula $\alpha$, there is a set of formulas $\circ(\alpha)$, uniquely determined by $\alpha$, such that for any set of formulas $\Gamma$ and for any formula $\beta, \Gamma \cup \circ(\alpha) \cup\{\alpha, \neg \alpha\} \vdash \beta$.

The principle (1) implies that LFIs are non-explosive logics, in the sense that a contradiction does not always entail arbitrary statements, i.e. the formula $\alpha \rightarrow(\neg \alpha \rightarrow \beta)$ is not valid. On the other hand, the intuitive motivation of (2) is that negation is only explosive with respect to consistent formulas; therefore a theory that admits $\circ(\alpha), \alpha, \neg \alpha$ is therefore trivial. This allows us to separate the sentences that make a theory explosive, from those that do not.

The weakest LFI is called $m b C$ and consists of positive classical propositional logic $\left(C P L^{+}\right)$, plus a paraconsistent negation $\neg$ and a consistency operator $\circ$ which are added, together with a minimal set of rules to make it an LFI. In this case $\circ(\alpha)$ is just the singleton $\{\circ \alpha\}$. Notice that the classical negation can be defined within $m b c$ as $\sim \alpha={ }_{d f}$. $\alpha \rightarrow(\circ \alpha \wedge \alpha \wedge \neg \alpha)$. Furthermore, $m b c$ constitutes a conservative extension of $C P L^{+}$, since every theorem of $C P L^{+}$can be recovered in $m b C$.

DEFINITION 8. The logic mbC is defined over the language the classical propositional language extended by the o-operator and is axiomatized by the following Hilbert-style axioms:

\section{Axiom schemas:}

$$
\begin{aligned}
& \alpha \rightarrow(\beta \rightarrow \alpha) \\
& (\alpha \rightarrow(\beta \rightarrow \gamma)) \rightarrow((\alpha \rightarrow \beta) \rightarrow(\alpha \rightarrow \gamma)) \\
& \alpha \rightarrow(\beta \rightarrow(\alpha \wedge \beta)) \\
& (\alpha \wedge \beta) \rightarrow \alpha \\
& (\alpha \wedge \beta) \rightarrow \beta \\
& \alpha \rightarrow(\alpha \vee \beta) \\
& \beta \rightarrow(\alpha \vee \beta) \\
& (\alpha \rightarrow \gamma) \rightarrow((\beta \rightarrow \gamma) \rightarrow((\alpha \vee \beta) \rightarrow \gamma)) \\
& \alpha \vee \neg \alpha \\
& \circ \alpha \rightarrow(\alpha \rightarrow(\neg \alpha \rightarrow \beta))
\end{aligned}
$$

Inference rule:

$$
\frac{\alpha \quad \alpha \rightarrow \beta}{\beta}(\mathbf{M P})
$$

Observe that $(\mathbf{A x} \mathbf{1}-\mathbf{A x} \mathbf{9})$ plus $(\mathbf{M P})$ constitute a Hilbert calculus over the signature for $C P L^{+}$, whereas (AX10) is called the gentle law of explosion and characterizes primarily $m b C$ as an LFI.

Next we will present the first order (with equality) version of $m b c$, the LFI $Q m b c_{\approx}$.

DEFINITION 9. Let $\Theta$ be a first-order signature with $a \approx-$ symbol for equality and that includes the o-operator. The logic $Q m b c_{\approx}$ is defined by a Hilbert Calculus that extends that for mbc over the signature $\Theta$, adding the following axiom schemas and inference rules: 


\section{Axiom schemas:}

$$
\begin{array}{lr}
\varphi[x / t] \rightarrow \exists x \varphi, \text { if } t \text { is a term free for } x \text { in } \varphi & (\mathbf{A x 1 1}) \\
\forall x \varphi \rightarrow \varphi[x / t], \text { if } t \text { is a term free for } x \text { in } \varphi & (\mathbf{A x 1 2}) \\
\alpha \rightarrow \beta, \text { whenever } \alpha \text { is a variant of } \beta & (\mathbf{A x 1 3}) \\
\forall x(x \approx x) & (\mathbf{A x} \mathbf{1 4}) \\
\forall x \forall y((x \approx y) \rightarrow(\alpha \rightarrow \alpha[x / y])), \text { if } y \text { is a variable free for } x \text { in } \alpha(\mathbf{A x 1 5})
\end{array}
$$

\section{Inference rules:}

$$
\begin{array}{ll}
\frac{\alpha \rightarrow \beta}{\alpha \rightarrow \forall x \beta} \text { if } x \text { is not free in } \alpha & (\forall-\text { In }) \\
\frac{\alpha \rightarrow \beta}{\exists x \alpha \rightarrow \beta} \text { if } x \text { is not free in } \beta & (\exists-\mathbf{I n})
\end{array}
$$

4.2 LFI-set theories. The basic system of paraconsistent set theory that we discuss are based on the logic $Q m b c_{\approx}$ and a first order signature $\Theta_{Z F}$, which contains, besides the equality predicate $\approx$, a binary predicate $\epsilon$ and a further unary predicate $C$, which stands for the consistency of sets - as opposed to formulas.

The possibility to distinguish between inconsistent and consistent sets is intended to capture a seminal intuition of Cantor, who made a clear separation between consistent and inconsistent totalities, that is, between sets and collections that are to big to be sets, like the totality of all alephs. This distinction was not only a terminological curiosity, since Cantor used the notion of inconsistent totality in a mathematical argument meant to show that the collection of cardinal is totally ordered by size; [13], p. 410.

DEFINITION 10. ([5]) The system ZFmbC is the first order theory with equality obtained from the logic $Q m b c_{\approx}$ over $\Theta_{Z F}$ by adding the following set- theoretic axiom schemata:

$$
\begin{aligned}
& \forall x \forall y[\forall z(z \in y \leftrightarrow z \in x) \rightarrow(x=y)] \text { (Extensionality) } \\
& \forall x \exists y \forall z(z \in y \leftrightarrow \forall w \in z(w \in x)) \text { (Power Set) } \\
& \forall x \exists y \forall z(z \in y \leftrightarrow \exists w \in x(z \in x)) \text { (Union) } \\
& \forall x \forall y \exists z \forall w(w \in x \leftrightarrow(w \approx x \vee w \approx y)) \text { (Pairing) } \\
& \left.\exists w\left(\left(\varnothing^{*} \in w\right) \wedge(\forall x)(x \in w \rightarrow x \cup\{x\} \in w)\right) \text { (Infinity }\right)^{8} \\
& \left.F U N_{\varphi} \rightarrow \exists b \forall y(y \in \leftrightarrow \exists x(x \in a \wedge \varphi(x, y))) \text { (Replacement }_{\varphi}\right)^{9} \\
& \left.(\exists b \forall x((x \in b) \leftrightarrow((x \in a) \wedge \varphi(x)))) \text { (Separation }_{\varphi}\right) \\
& C(x) \rightarrow(\exists y(y \in x) \rightarrow \exists((y \in x) \wedge \sim \exists z(z \in x \wedge z \in y))) \text { (Weak regularity) } \\
& (x \not \approx y) \leftrightarrow \exists z((z \in x) \wedge(z \in y)) \vee \exists z((z \in y) \wedge(z \in x)) \text { (Unextensionality) } \\
& \forall x\left(x \in y \rightarrow(C(x) \rightarrow C(y))\left(\mathbf{C o n}_{\mathbf{0}}\right)\right. \\
& \forall x(C(x) \rightarrow \circ(x=x))\left(\mathbf{C o n}_{1}\right) \\
& \forall x \neg \circ(x=x) \rightarrow \neg C(x)\left(\mathbf{C o n}_{2}\right)
\end{aligned}
$$

Notice that in ZFmbC we have at disposal a classical negation and a paraconsistent one $\neg$, since this is already the case in $Q m b C_{\approx}$. Moreover, we can use $x \not \approx y$ to abbreviate $\neg(x \approx y)$ and $x \notin y$ to abbreviate the formula $\neg(x \in y)$. Notice that the first six axioms,

\footnotetext{
${ }^{8}$ In the axiom of infinity $\varnothing^{*}$ stands for the strong empty set $\varnothing^{*}:=\{x \mid \sim(x \approx x)\}$.

${ }^{9}$ For the replacement schema let $\varphi(x, y)$ be a formula where variables $x$ and $y$ occur free. Let $z$ be a fresh variable. Then $F U N_{\varphi}$ denotes the following formula: $F U N_{\varphi}=_{D f} \forall x \forall y \forall z(\varphi(x, y) \wedge \varphi(x, z) \rightarrow$ $(y \approx z)$.
} 
when added to classical first order logic with identity together with the regular regularity axiom constitute the ZF set theory. Therefore the non classical flavor of this set theory stems from Weak regularity, Unextensionality and the axioms governing the consistency predicate.

In [5] it is proved that ZFmbC is non-trivial provided that $\mathrm{ZF}$ is consistent. As a consequence, this paraconsistent set theory — as it is the case in $V^{\mathbb{P S}_{3}}$-does not allow the Russel set in their respective ontology. On the other hand this set theory is extremely weak with regard to its expressive power, since we can not define inconsistent sets nor the inconsistency operator. To this aim, we need to consider extensions of ZmbC defined by taking stronger LFIs and appropriate axioms for the consistency predicate. The first system we consider is based on the logic $\mathrm{mCi}$.

Definition 11. ([5]) The system ZFmCi is obtained from ZFmbC by adding the following axioms, for $n \geqslant 0$ :

$$
\begin{gathered}
\neg \circ \varphi \rightarrow(\varphi \wedge \neg \varphi)(\mathbf{c i}) \\
\neg^{n+2} \circ \varphi \rightarrow \neg^{n} \circ \varphi\left(\neg^{n}\right) \\
\forall x\left(\neg C(x) \rightarrow \neg \circ(x \approx x)\left(\mathbf{C o n}_{3}\right)\right. \\
\forall x\left(\neg C(x) \rightarrow \neg \circ(x \in x)\left(\mathbf{C o n}_{4}\right)\right.
\end{gathered}
$$

The first two axioms (ci) and $\left(\neg^{n}\right)$ transform the underlying logic $m b C$ into the stronger logic $m C i$, in which the inconsistency operator can be defined as $\bullet \varphi:=\neg \circ \varphi$ and inconsistent sets as the dual of consistents sets, so $I(x):=\neg C(x)$.

DEFINITION 12. The system ZFCi is obtained from ZFmCi by adding axiom $\mathbf{c f}$ :

$$
\neg \neg \varphi \rightarrow \varphi(\mathbf{c f})
$$

DEFINITION 13. Let ZFCil be the system obtained from ZFmCi by adding the following axioms:

$$
\begin{gathered}
\neg(\varphi \wedge \neg \varphi) \rightarrow \circ \varphi(\mathbf{c l}) \\
\forall x((\neg(x \approx x) \wedge(x \not z x)) \rightarrow C(x))\left(\mathbf{C o n}_{\mathbf{5}}\right) \\
\forall x((\neg(x \in x) \wedge(x \notin x)) \rightarrow C(x))\left(\mathbf{C o n}_{\mathbf{6}}\right)
\end{gathered}
$$

\section{LFI-set theories and linear models}

In this section we will show that the paraconsistent Zermelian set theories ZFmbC, $\mathrm{ZFmCi}, \mathrm{ZFCi}$ and $\mathrm{ZFCil}$ are not valid in any $\mathfrak{B}^{*}$ - valued model ( we will refer to them simply as linear models). This result is counterintuitive, since by Theorem 4 the threeelements linear algebra is nothing else then $\left(\mathbb{P S}_{3}, *\right)$, which is an LFI, as noted in [7]. This is odd and will reveal serious issues in the conception and motivation of these Zermelian set theories.

5.1 ZF-axioms. In order to check whether the axioms of an LFI-set theory is valid in a linear model, we first need to specify the interpretation of the o-operator. Since this operator is the source of classicality, we use the following evaluation $\llbracket \circ \varphi \rrbracket=\llbracket \varphi \rightarrow \perp \rrbracket \vee \llbracket \sim$ $\varphi \rightarrow \perp \rrbracket$, which expresses the fact that $\varphi$ is consistent if either $\varphi$ or its negation is false. In the three element case this gives rise to the following table. 


\begin{tabular}{|c|c|}
\hline$\varphi$ & $\circ \varphi$ \\
\hline 1 & 1 \\
$\frac{1}{2}$ & 0 \\
0 & 1 \\
\hline
\end{tabular}

Figure 3. Three valued matrix for consistency operator

We now check the validity of the axioms of ZFmbC in the linear models. Since the focus of this work is paraconsistency we require our models to be non-classical.

DeFinition 14. By a paraconsistent linear algebra we mean a $\mathfrak{V}$-algebras $\mathbb{L}$ such that $|\mathbb{L}|>2$. The class of such algebras will be indicated by $\mathfrak{V}^{*}$ and its elements $\mathfrak{V}^{*}$-algebras. By a paraconsistent linear model we mean a $\mathfrak{V}$-model of the form $\mathbf{V}^{\mathbb{L}}$, with $|\mathbb{L}|>2$, and we refer to it as a $\mathfrak{V}^{*}$-model.

By Theorem 4 we know that the negation free fragment of ZF is valid in any linear model. Therefore, any linear model verifies (Union), (Extensionality), (Power set), $\left(\right.$ Separation $\left._{\varphi}\right),\left(\text { Foundation }_{\varphi}\right)^{10}$, and $\left(\right.$ Replacement $\left._{\varphi}\right)$, for $\varphi$ negation free.

On the other hand, (Infinity) and (Weak regularity), as presented in [4], are not valid in any $\mathfrak{V}^{*}$-model. Nonetheless, by a suitable modification we can obtain their validity, without distorting the spirit of ZFmbC. The issue with (Infinity) consists in the use of the strong empty set $\varnothing^{*}$ which cannot be defined in the $\mathfrak{V}^{*}$-models. In [4], the authors define the strong empty set using (Separation) and (Extensionality) and the fact that $\mathrm{ZFmbC} \vdash((x \in a) \wedge \sim(x=x)) \leftrightarrow \sim(x=x)$. However, this cannot be done in any $\mathfrak{V}^{*}$-model, since the formula that defines the strong empty set is not a negation free instantiation of separation. But this is not dramatic, since we can use a different, negation free, formulation of (Infinity) that suits better an algebra-valued model treatment of set theory: $:^{11}$

$$
\exists x[\exists y(\forall z(z \in y \rightarrow \perp) \wedge y \in x) \wedge \forall w \in x \exists u \in x(w \in u)]
$$

On the other hand, (Weak regularity) states that only consistent sets cannot be sets of themselves. But notice that (Foundation $_{\varphi}$ ) is valid in any $\mathfrak{V}^{*}$-model, for $\varphi$ negation free and therefore we have that $\llbracket x \in x \rrbracket^{\mathbb{L}}=0$, for all $x \in \mathbf{V}^{\mathbb{L}}$ and $\mathbb{L} \in \mathfrak{V}$. Thus even inconsistent sets cannot be ill-founded. This seems to suggest that we should discard (Weak regularity) in favor of (Foundation For , for negation free.

For what concerns (Unextensionality) the situation is more serious. Before stating the relevant result, a word on notation. When we say that an algebra-valued model satisfies a formula we mean that, for any filter we can define on the algebra, the evaluation will yields a value in the set of designated values. In this case we write $\mathbf{V}^{\mathbb{L}} \models \varphi$. On the contrary, if we want to stress the selected filter we use the following notation. Given a filter $G \subseteq A$ defined on the base set of an algebra $\mathbb{A}$ we write $\mathbf{V}^{\mathbb{L}} \models_{G} \varphi$ to say that $\varphi$ is valid in $\mathbf{V}^{\mathbb{L}}$ according to the consequence relation engendered by the filter $G$.

THEOREM 15. Let $\mathbf{V}^{\mathbb{L}}$ be a $\mathfrak{V}^{*}$-model, then $\mathbf{V}^{\mathbb{L}} \not=$ (Unextensionality).

ProOF. We prove that $\mathbf{V}^{\mathbb{L}} \not \models$ (Unextensionality) by showing that there is a filter $G \subseteq L$ such that $\mathbf{V}^{\mathbb{L}} \not \models_{G}($ Unextensionality $)$. Notice that $\left(\mathbb{P S}_{3},{ }^{*}\right)$ is contained in any

${ }^{10}$ Foundation is here intended in terms of transfinite recursion, which is indeed a schema

11 This definition of the axiom of infinity was already proposed in [1]. 
$\mathfrak{V}^{*}$-algebra. Let us call the elements of the base set of $\left(\mathbb{P S}_{3},{ }^{*}\right)$ as $\left\{0, \frac{1}{2}, 1\right\}$. And let define $G$ to be a filter that contains $\frac{1}{2}$, and hence all the elements above $\frac{1}{2}$.

We can consider the following names as witnesses for all $\mathrm{V}^{*}$-models: $p_{\frac{1}{2}}=\left\langle\varnothing, \frac{1}{2}\right\rangle, x=$ $\left\langle p_{\frac{1}{2}}, 1\right\rangle, y=\left\langle p_{\frac{1}{2}}, \frac{1}{2}\right\rangle$. Now, we simply calculate the value of (Unextensionality) in a generic $\mathfrak{V}^{*}$-model $\mathbf{V}^{\mathbb{L}}$ :

$$
\begin{aligned}
& =\bigvee_{w \in \mathbf{V}^{\mathbb{L}}}(\llbracket w \in x \rrbracket \wedge \llbracket w \in y \rrbracket) \vee \bigvee_{w \in \mathbf{V}^{\mathbb{L}}}(\llbracket w \in x \rrbracket \wedge \llbracket w \in y \rrbracket) \\
& \quad=\left(1 \wedge \frac{1}{2}\right) \vee\left(1 \wedge \frac{1}{2}\right)
\end{aligned}
$$

Now we can deduce the truth values of $\llbracket w \in y \rrbracket^{*}=\frac{1}{2}$ and $\llbracket w \in x \rrbracket^{*}=0$. Furthermore, it is easy to show that $\llbracket x=y \rrbracket=1$ and by Lemma $6 \llbracket \neg(x=y) \rrbracket=\llbracket x=y \rrbracket^{*}=0$. Now, we can calculate readily

$$
\bigvee_{w \in \mathbf{V}^{\mathbb{L}}}\left(\llbracket w \in x \rrbracket \wedge \llbracket w \in y \rrbracket^{*}\right) \vee \bigvee_{w \in \mathbf{V}^{\mathbb{L}}}\left(\llbracket w \in x \rrbracket^{*} \wedge \llbracket w \in y \rrbracket\right)=\frac{1}{2}
$$

Since we know that the antecedent of the unextensionality axiom receives truth value $\frac{1}{2}$ and the consequent truth value 0 , we can conclude by Corollary 3.11 that the right-to-left implication of (Unextensionality) and therefore the axiom itself, receives truth value 0 .

Notice that the above argument can be adapted to show that, given a $\mathfrak{V}^{*}$-model $\mathbf{V}^{\mathbb{L}}$ and a filter $G \subseteq L$ such that $|G|>2$, there are always three elements in $\mathbf{V}^{\mathrm{L}}$ that witness the failure of (Unextensionality). It is sufficient to choose the relevant intermediate element of $\mathbb{L}$ - that plays the role of $\left.\frac{1}{2}\right)$ in $\left(\mathbb{P S}_{3},{ }^{*}\right)$ - high enough to be in the filter. ${ }^{12}$ The case $|G|=1$ is not relevant since in this case $G=\{1\}$ and therefore the resulting consequence relation is classical.

Therefore (Unextensionality) fails very badly in any $\mathfrak{V}^{*}$-model, disregarding the choice of the consequence relation. This is already troubling, but a possible strategy would consist in biting the bullet and just to eliminate this axioms. However, we will show that the issues of LFI-set theories lay much deeper in the interpretation of inconsistent sets.

5.2 C-axioms. In [5] the definition of inconsistency is given in terms of the violation of the basic logical operations of set theory: equality and membership.

(i) $\neg C(x) \leftrightarrow(x \in x)$

(ii) $\neg C(x) \leftrightarrow \neg \circ(x \in x)$

(iii) $\neg C(x) \leftrightarrow(x \not \approx x)$

(iv) $\neg C(x) \leftrightarrow \neg \circ(x \not \approx x)$

We now show that these definitions cannot capture the notion of inconsistency in any linear model.

12 Notice that it is in this part of the argument that we use Lemma 6 , to show that an intermediate value is mapped to itself by the *-negation. 
Proposition 16. For $\mathbb{L} \in \mathfrak{V}^{*}$, we have that (i) $\llbracket x \in x \rrbracket^{\mathbb{L}}=0$, (ii) $\llbracket \neg \circ(x \in x) \rrbracket^{\mathbb{L}}=0$, (iii) $\llbracket x \not x \rrbracket^{\mathbb{L}}=0$ and (iv) $\llbracket \neg \circ(x \not \approx x) \rrbracket^{\mathbb{L}}=0$.

Proof. Notice that we have already argued that $\left(\right.$ Foundation $\left._{\varphi}\right)$, for $\varphi$ negation free, yields $\llbracket x \in x \rrbracket^{\mathbb{L}}=0$. This establishes (i). From (i) we know that $\llbracket x \in x \rrbracket^{\mathbb{L}}$ receives a classical truth value. Hence we deduce $\llbracket \circ(x \in x) \rrbracket^{\mathbb{L}}=1$. Then by Lemma $6 \llbracket \neg \circ(x \in$ $x) \rrbracket^{\mathbb{L}}=0$, establishing (ii). Adapting Proposition 1 of [9], we know that for every $x \in \mathbf{V}^{\mathbb{L}}$ $\llbracket x \approx x \rrbracket^{\mathbb{L}}=1$ and $u(x) \leqslant \llbracket x \in u \rrbracket$ for every $u$ in the domain of $x$, so $\llbracket x \not x \rrbracket^{\mathbb{L}}=0$. From this follows (iii). Furthermore, given the classical truth value of $\llbracket(x \not \approx x) \rrbracket^{\mathbb{L}}$ it follows that $\llbracket \circ(x \not \approx x) \rrbracket^{\mathbb{L}}=1$. Finally, given Lemma 6 we can conclude $\llbracket \neg \circ(x \not \approx x) \rrbracket^{\mathbb{L}}=0$, which settles (iv).

This proposition shows clearly that none of the above interpretations of inconsistency can ever be realized in any $\mathfrak{V}^{*}$-model.

\section{Conclusion}

The failure of the characteristic axioms of these LFI-set theories, together with the impossibility to define inconsistency in the way proposed in [5], leaves us with two options. Either we assume that, $\forall x \in \mathbf{V}^{\mathbb{L}} \llbracket \neg C(x) \rrbracket^{\mathbb{L}}=0$, therefore assuming that every set is consistent, or we can try to modify the interpretation of the consistency predicate in order to provide examples of inconsistent sets in $\mathfrak{V}^{*}$-models. We argue that both strategies are unfeasible.

Let us make explicit the two horns of this dilemma:

Horn 1: The first strategy consists in eliminating inconsistent totalities from the picture. This option is, as a matter of fact, not very far from Cantor's ideas. Indeed in several letters to Hilbert and Jourdain ([13], p.425-435) Cantor identified sets and consistent totalities, arguing that the universe of set was the collection of all totalities that did not lead to any contradiction. On this basis we can therefore postulate an axiom, that we can call Cantorian Axiom, that expresses this intuition.

$$
C(x) \leftrightarrow \operatorname{Set}(x) \quad \text { (Cantorian Axiom) }
$$

Now, if we extend ZFmbC with the Cantorian Axiom we obtain that all $\mathfrak{V}$-models validate this set theory and all its extension. Indeed these LFI-set theories would all be equal ZF, since we have just removed all inconsistent sets. However, this move would totally trivialize the main motivation of using a consistency predicate in dealing with an inconsistent set theory, as clearly expressed [4], p. 366.

The main idea is to assume that not only sentences can be taken to be consistent or inconsistent, but also that sets themselves can be thought to be consistent or inconsistent. We establish the basis for new paraconsistent set-theories (such as ZFmbC and ZFCil) under this perspective and establish their non-triviality, provided that $\mathrm{ZF}$ is consistent.

Horn 2: On the other hand, we could try to modify the interpretation of the (in)consistency predicate, in order to have at least one set $x$ such that $\llbracket \neg C(x) \rrbracket \geqslant a$, for a designated value $a \neq 1$ of a $\mathfrak{V}^{*}$-algebra. However, also this strategy is doomed to fail, since no $\mathfrak{V}^{*}$-model would be able to validate $\mathrm{ZFmCi}$, or $\mathrm{ZFCi}$, or ZFCil.

LEMMA 17. Let $\mathbf{V}^{\mathbb{L}}$ be a $\mathfrak{V}^{*}$-model, $G \subseteq L$ a filter containing an element $a \in L$ that is different from 1 , and $x \in \mathbf{V}^{\mathbb{L}}$ such that $\llbracket \neg C(x) \rrbracket^{\mathbb{L}} \geqslant a$. Then $\mathbf{V}^{\mathbb{L}} \not \models \neg C(x) \rightarrow \neg \circ(x=x)$ 
Proof. Due to Proposition 1 in [9], we know that $\llbracket \circ(x=x) \rrbracket^{\mathbb{L}}=1$ and therefore $\llbracket \neg \circ(x=x) \rrbracket^{\mathbb{L}}=0$. Then by Corollary 3.11 :

$$
\llbracket \neg C(u) \rrbracket^{\mathbb{L}} \geqslant a \rightsquigarrow \llbracket \neg \circ(u=u) \rrbracket^{\mathbb{L}}=0 .
$$

Notice that in this case we would validate (vacuously) all the axioms of ZFmbC that govern the consistency predicate, with exception of $\mathbf{c o n}_{\mathbf{0}}$. This is due to the fact that either $\neg C(x)$ occurs in the consequent of an axiom, so that the antecedent is always false, as it is the case with $\mathbf{c o n}_{2}$, or $C(x)$ occurs in the antecedent, so that the consequent will be trivially true, as in $\mathbf{c o n}_{1}$.

Besides the formal problems that arise from accepting the existence of inconsistent totalities, there is also a deeper conceptual issue. A charitable interpretation of the inconsistency predicate - that seems faithful to the history of set theory - would consists in equating inconsistent objects and proper classes. However, at a closer look the axioms of ZFmbC force us to discard this possibility, since the axiom $\mathbf{c o n}_{0}$ would implies that the universal class is a set and not a proper class. Indeed, it says that the property of being a set is propagated $\in$-upward, which clearly cannot be the case since all elements of $\mathbf{V}$ are, by definitions, sets.

We can therefore conclude, from Horn 1 and Horn 2, that the LFI-set theory we discussed are not valid in the most natural models for Zermelian set theories at disposal. This is even more belittling, considering that the internal logic of $\mathbf{V}^{\left(\mathbb{P S}_{3},{ }^{*}\right)}$ is an LFI. Not only we need to give up the characteristic axiom (Unextensionality), but Horn 2, together with the charitable interpretation of inconsistent totalities as classes, shows that not even the weak ZFmbC-axioms that govern the $C$-predicate can hold in a $\mathfrak{V}^{*}$-model. We believe that the fact that inconsistent totalities do not find a place in these LFI-set theories is a serious betrayal of their original motivation, which therefore suggest a fresh new start. The failure of this attempt leaves open the challenging problem of making inconsistent totalities compatible with a Zermelian set theory based on a paraconsistent logic. We leave this open question for future work.

\section{Acknowledgements}

The first author acknowledges support from the FAPESP grant. n. 2017/23853-0. The second author acknowledges support from the FAPESP Jovem Pesquisador grant n. 2016/25891-3. 


\section{Bibliography}

[1] J. Bell. Set theory. Boolean valued models and independence proofs. Oxford Science Pubblications, 2005.

[2] George Boolos. The Iterative Conception of Set. The Journal of Philosophy, 68(8):215-231, April 1971.

[3] Ross T. Brady. The non-triviality of dialectical set theory. In G. Priest, R. Routley, and J. Norman, editors, Paraconsistent Logic: Essays on the Inconsistent, pages 437-470. Philosophia Verlag, 1989.

[4] Walter Carnielli and Marcelo E. Coniglio. Paraconsistent Logic: Consistency, Contradiction and Negation. Basel, Switzerland: Springer International Publishing, 2016.

[5] Walter Carnielli and Marcelo E. Coniglio. Paraconsistent set theory by predicating on consistency. Logique Et Analyse, 26(1):97-116, 2016.

[6] Paul Cohen. The independence of the continuum hypothesis. Proc. Nat. Acad. Sci. USA, 50(6):1143-1148, 1963.

[7] M. E. Coniglio and L. H. D. C. Silvestrini. An alternative approach for quasi-truth. Logic Journal of the IGPL, 22(2):387-410, 2014.

[8] Newton Ca da Costa. On paraconsistent set theory. Logique Et Analyse, 115:361-371, 1986.

[9] Benedikt Löwe and Sourav Tarafder. Generalized algebra-valued models of set theory. Review of Symbolic Logic, 8(1):192-205, 2015.

[10] João Marcos. 8K solutions and semi-solutions to a problem of da Costa. Unpublished.

[11] João Marcos. Logics of Formal Inconsistency. PhD Thesis.

[12] Santiago J. Martinez and Giorgio Venturi. Non-classical Models of ZF. Studia Logica, 2020. DOI: https://doi.org/10.1007/s11225-020-09915-0. (Updated on July 11, 2020).

[13] W. Nilson and H. Meschkowski. Georg Cantor. Briefe. Springer Verlag, 1991.

[14] Greg Restall. A note on naive set theory in $\$\{\mathrm{Rm}$ LP $\} \$$. Notre Dame Journal of Formal Logic, 33(3):422-432, 1992.

[15] Zach Weber. Notes on inconsistent set theory. In Francesco Berto, Edwin Mares, Koji Tanaka, and Francesco Paoli, editors, Paraconsistency: Logic and Applications, pages 315-328. Springer, 2013. 